\title{
An Improved DC Fault Protection Scheme Independent of Boundary Components for MMC based HVDC Grids
}

\author{
Saizhao Yang, Wang Xiang, Member, IEEE, Jinyu Wen, Member, IEEE
}

\begin{abstract}
For modular multilevel converter (MMC) based DC grids, current-limiting reactors (CLRs) are mainly employed to suppress the fault current and provide boundary effects to detect internal faults. Thus, most existing protection schemes are highly dependent on the larger CLRs to guarantee high selectivity. However, in existing MMC based HVDC projects, the size of CLRs is restrained by the cost, weight, and system stability under normal state. Thus, boundary protections may fail to detect high-resistance faults and pole-to-ground faults under weak boundary conditions. To overcome these shortcomings, this paper proposes a fast and selective DC fault detection scheme independent of boundary components. The propagation characteristics of line-mode backward traveling-waves (TW) are analyzed to identify external and internal faults. The polarities of zero-mode backward TWs are employed to select faulted poles. The directional overcurrent based pilot protection is adopted as a complementary criterion to detect remote faults. The proposed method can be applied in MMC-HVDC systems with small CLRs that cannot provide strong boundary conditions. Besides, the detection speed is fast (less than $\mathbf{1 . 5 m s}$ ). Moreover, it is robust to fault resistance and immune to noise. Various simulation results in PSCAD/EMTDC verifies the effectiveness of the proposed scheme.
\end{abstract}

Index Terms - backward traveling wave, boundary components, current limiting reactor, DC fault detection, HVDC grids, MMC.

\section{INTRODUCTION}

The modular multilevel converter (MMC) based DC grids have promising advantages on the asynchronous grid interconnection, bulk renewable energy integration over long distance and power supply for passive systems, which have drawn significant attention in both industry and academia [1][2]. When DC grids are subject to pole-to-pole faults, submodule capacitors of converters will discharge into the fault point quickly, resulting in high fault currents with fast-rising speed. To ensure the safe operation of the system, a fast and selective fault detection algorithm is required to isolate faulted lines. Taking the world's first $\pm 500 \mathrm{kV}$ Zhangbei DC grid as an example, internal faults are required to be selectively identified within $3 \mathrm{~ms}$, which brings about great challenges to guarantee the reliability and sensitivity of fault protection [3].

To avoid damage to power electronic devices during DC

This work is sponsored by the Joint Funds of the National Natural Science Foundation of China (U1766211) and the China Postdoctoral Science Foundation (2019M662622). (Corresponding author: Wang Xiang)

S. Yang, J. Wen are with the State Key Laboratory of Advanced Electromagnetic Engineering and Technology, Huazhong University of Science and Technology, Wuhan 430074, China. (e-mail: saizhaoyang@foxmail.com, jinyu.wen@ @ust.edu.cn).

W. Xiang is with the Department of Electronic and Electrical Engineering, University of Strathclyde, Glasgow, G1 1XW, U.K. (e-mail: xiangwang1003@foxmail.com). fault detection, current-limiting reactors (CLR) are implemented on the transmission lines to suppress the fault current rise rate [4]. Due to the smoothing effect of CLRs, the steep wave-front of fault traveling-wave (TW) induced from the fault point will be attenuated and the high-frequency components of DC voltage/current will be "filtered" by CLRs, which provides a boundary effect to identify internal and external faults. Considering this boundary effect, existing protection schemes can be divided into three categories: I) time-domain based TW methods[5]-[7], II) frequency-domain or Wavelet Transform (WT) based methods[8]-[12], III) reactor voltage based methods [4][13][14]. Among these methods, time-domain based TW methods are ineffective to fault resistances and vulnerable to noise. To improve the robustness to fault resistance, WT is employed to extract high-frequency components or identify the arrival of the fault TW. For the third category, a reactor voltage-based method using modal domain analysis is proposed to overcome the decencies of disability to detect pole-to-ground (PTG) faults and weakness to fault resistance [4].

The reliability and selectivity of the aforementioned protection algorithms based on boundary effects are highly dependent on large CLRs. As pointed out in [15], for existing protection schemes, large CLRs (over $100 \mathrm{mH}$ ) are adopted to enable high selectivity and reliability. However, there are several scenarios where the configured current-limiting inductors are small and cannot provide strong boundary conditions for fault protection algorithms. 1) In existing cable-based MMC-HVDC projects, the sizes of CLRs implemented at the terminal of cables are small, due to the little likelihood of DC fault occurrence. For example, $50 \mathrm{mH}$ CLRs are implemented in the $\pm 320 \mathrm{kV}$ INELFE MMC-HVDC project which interconnects national electrical grids between France and Spain[16]; 20mH and 10mH CLRs are adopted in the $\pm 200 \mathrm{kV}$ Zhoushan five-terminal MMC-HVDC project and the $\pm 160 \mathrm{kV}$ Nan'ao three-terminal MMC-HVDC project, respectively. 2) Fault tolerable converter based HVDC systems. Due to the current-limiting capability of the converter, there is no need to implement large CLRs. For example, the Kunliulong three-terminal HVDC project constructed by hybrid MMC will use a CLR of $100 \mathrm{mH}$ for fault current limiting [17]. Besides, on account of manufacturing difficulties, a large CLR will be separated into two smaller ones, implementing at the polar and ground lines respectively, to reduce the volume and weight of one single inductor. Taking Zhangbei DC grid project as an example, to avoid blocking converters during DC faults, a 450mH CLR is required. Where 150mH CLRs are installed at the positive and negative polar lines respectively and 300mH CLRs are installed on the ground lines [18]. For the CLRs on the ground lines, they cannot provide boundary effects for the boundary 
protection schemes [18]. It can be seen that CLRs implemented on polar lines are no more than $150 \mathrm{mH}$ in existing MMC-HVDC projects. Thus, protection schemes in [3][7][9][10][13] which rely on 200mH CLRs may not be applicable to existing MMC-HVDC projects. To be concluded, a reliable protection algorithm independent of CLRs is required to guarantee high selectivity under weak boundary conditions.

Pilot protection schemes can guarantee high reliability to identify external and internal faults without depending on boundary effects [19]-[20]. However, for close-in faults that bring about higher fault current, the pilot protection schemes suffer from slow detection speed. To improve the fault detection speed, a single-end protection scheme independent of CLRs is proposed in [21]. The differences in the arrival times between line-mode and zero-mode TWs are employed to identify internal faults selectively and quickly. However, due to the inexistence of zero-mode TW, the method in [21] cannot detect pole-to-pole (PTP) faults.

To overcome the aforementioned challenges, an improved DC fault protection algorithm independent of boundary components is proposed for MMC based DC grids in this paper. The relationship between the line-mode backward TW propagation characteristics and the fault distance is analyzed, thereby designing the fault detection criterion as the Zone I primary protection. The polarities of zero-mode backward TW are employed to design the faulted pole selection criterion. Besides, the directional overcurrent based pilot protection is adopted as the Zone II primary protection to detect remote faults with fast detection speed and high reliability. The contributions of this paper are as follows:

1) Compared with existing boundary protection schemes, the proposed method does not need large CLRs to provide boundary effects. Thus, the cost and weight of CLRs can be reduced while still maintain sufficient current-limiting capability.

2) Compared with existing non-boundary protection schemes, the proposed protection scheme is robust to fault resistances and fault types and improve the reliability under weak boundary effects.

3) The TW propagation characteristic is adopted to design the Zone I primary protection, which is highly determined by the fault distance rather than the fault resistance. Thus, compared with some time-domain TW based methods, the proposed method is robust to fault resistances. In addition, the Zone II directional overcurrent based pilot protection together with the Zone I primary protection is adopted to protect the entire transmission line with fast detection speed.

The remainder of the paper is structured as follows. Section II analyzes the impacts of boundary effects on protection methods and the speediness of pilot protection. The propagation characteristic analysis of backward TW is conducted in Section III. Then, the overall protection scheme is illustrated in Section IV. The effectiveness and sensitivity of the proposed method are verified in Sections V and VI. The rationality of using the directional overcurrent pilot protection as the Zone II primary protection is evaluated and the configuration of redundant primary protection schemes is discussed in Section VII. Finally, the conclusion is drawn in Section VIII.

\section{LIMITATION OF EXISTING BOUNDARY PROTECTION}

A. Impacts of Boundary Components on Boundary Protections

For reactor voltage based protection methods, impacts of boundary effects have been discussed in [4][13][14]. Concerning protection methods of the other two categories, i.e. the time domain based TW methods and the WT-based methods, the impacts of CLRs on typical fault protection methods, such as rate of change of voltage (ROCOV) [5], transient voltage (TV) [8] will be analyzed as follows.

\section{1) Impacts of CLRs on ROCOV [5] (protection category I)}

In the case of DC fault on the transmission line, the fault voltage wave induced from the fault point propagates along the transmission line towards the terminals. Assuming that the direction from the converter to the transmission line is positive, the detected initial voltage TW at terminal $\mathrm{A}$ is treated as backward TW $V_{b}$, as shown in Fig.1.

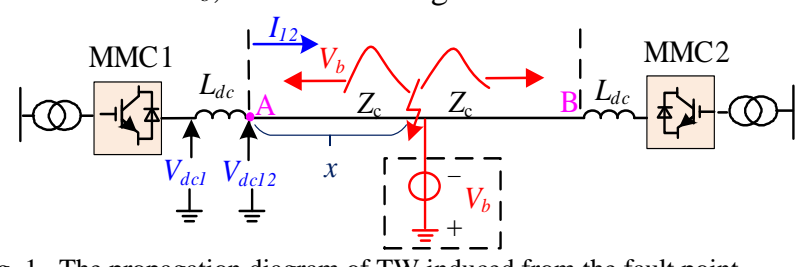

Fig. 1. The propagation diagram of $\mathrm{TW}$ induced from the fault point

When the negative TW $V_{b}$ arrives at terminal A, the DC line voltage $V_{d c 12}$ rapidly decreases. Meantime, the TW will be reflected and refracted. Ignoring the attenuation effects of the transmission line, the changes of MMC output DC voltage $V_{d c l}$ and DC line voltage $V_{d c 12}\left(\Delta V_{d c l}\right.$ and $\left.\Delta V_{d c 12}\right)$ can be expressed as:

$$
\Delta V_{d c l}=\alpha_{1} V_{b} e^{-\frac{t}{T_{L}}} ; \Delta V_{d c l 2}=V_{b}
$$

where $\alpha_{I}$ is the refraction coefficient at terminal $\mathrm{A}$ and $T_{L}$ is the time constant:

$$
T_{L}=\frac{L_{d c}}{Z_{M M C}+Z_{C}} \quad ; \quad \alpha_{1}=\frac{2 Z_{M M C}}{Z_{M M C}+Z_{C}}
$$

where $Z_{C}$ and $Z_{M M C}$ represent the wave impedance of transmission lines and the equivalent impedance of converter respectively. $L_{d c}$ is the current-limiting reactor.

As can be seen from equations (1)(2), due to the smoothing effects of CLRs, the sharp wave-front of initial TW $V_{b}$ will be attenuated, leading to a slow decrease of voltage $V_{d c l}$. Thus, the amplitude of the change rate of $V_{d c l}$ is much smaller than that of $V_{d c 12}$. Besides, the larger CLR (i.e. $L_{d c}$ ) will cause greater differences between the change rate of $V_{d c 1}$ and $V_{d c 12}$, which demonstrates that ROCOV is dependent on large CLRs to guarantee the selectivity.

2) Impacts of CLRs on TV [8](protection category II)

Due to multiple reflections and refractions of TWs, there exist high-frequencies in voltage $V_{d c 12}$. Based on the equivalent circuit of $\mathrm{MMC}$ that ignores the resistance in [4], the transfer function $\left|V_{d c l} / V_{d c 12}\right|$ that represents the line boundary effects in frequency-domain can be expressed as: 


$$
\left|\frac{V_{d c l}}{V_{d c l 2}}\right|=\left|\frac{Z_{M M C}(\omega)}{2 Z_{d c}(\omega)+Z_{M M C}(\omega)}\right|=\left|\frac{\frac{2 j \omega L}{3}+\frac{N}{6 j \omega C}}{2 j \omega L_{d c}+\frac{2 j \omega L}{3}+\frac{N}{6 j \omega C}}\right|
$$

where $L$ and $C$ are the arm inductance and sub-module of MMC capacitance respectively.

Using parameters in Table 1, the characteristics between amplitude and frequency of $\left|V_{d c l} / V_{d c 12}\right|$ can be obtained, as shown in Fig. 2.

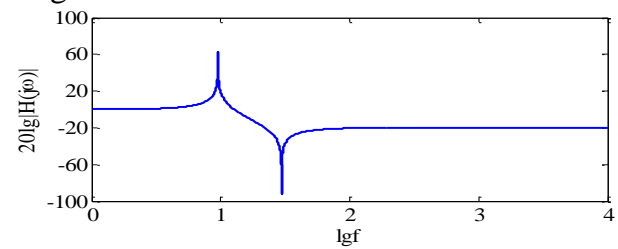

Fig. 2. The characteristics between amplitude and frequency of $\left|V_{d c} / V_{d c l 2}\right|$.

In Fig. 2, it can be concluded that $V_{d c l 2}$ possesses plentiful high-frequency components, while $V_{d c l}$ has few high-frequency components due to the attenuation effect by CLRs. In the case of a smaller CLR, the high-frequency differences between transient voltages $V_{d c l 2}$ and $V_{d c l}$ will become weaker, leading to the reduced reliability of the TV-based protection.

In summary, the aforementioned protection methods are highly dependent on boundary effects. With the decrease of current limiting inductance, the accuracy of the protection methods will deteriorate. Hence, a fast and reliable protection scheme independent of boundary components is required.

\section{B. The Speediness Analysis of Pilot Protection}

Taking the line protection relay 12 as an example, Fig. 3 shows the diagram of the detection time of pilot protection schemes. Where $x$ represents the fault distance and $l$ represents the total length of the transmission line. $t_{l}$ is the fault TW propagation time from the fault point to the opposite terminal. $t_{2}$ is the signal transmission time from the relay 21 to the relay 12. Without regard to the time window $\Delta t$ for fault characteristic capture, the detection time for pilot protection can be calculated as:

$$
t_{d}(\text { for pilot protection })=\frac{l-x}{v_{T W}}+\frac{l}{v_{o f}}
$$

where $v_{T W}$ represents the line-mode TW propagation velocity of the overhead line (OHL) and it is close to $3 \times 10^{5} \mathrm{~km} / \mathrm{ms}$. $v_{\text {of }}$ represents the velocity of light in fiber optical and it is $2 \times 10^{5}$ $\mathrm{km} / \mathrm{ms}$ [22]. For single-end protection schemes, the detection time can be calculated as:

$$
t_{d}(\text { for single-end protection })=\frac{x}{v_{T W}}
$$

Compared with the single-end protection, the extra time delay for pilot protection can be calculated as:

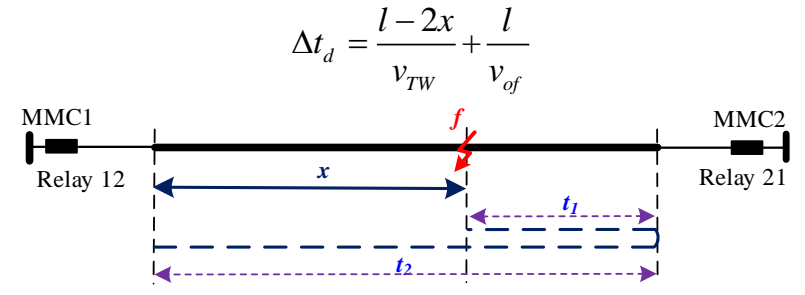

Fig. 3. The diagram of the detection time of pilot protections.

As can be seen from equations (4)-(6), for relay 12, when fault distance is smaller, the detection delay of pilot protection is longer compared with single-end protection. It indicates that pilot protection methods are only suitable to detect remote faults. Thus, a fast single-end protection scheme independent of boundary components is necessary to guarantee fast and reliable fault detection.

\section{The Theoretical ANALYsis of Protection SCHEME BASED ON BACKWARD TW}

\section{A. Analysis of TW Propagation Characteristics}

As shown in Fig.1, once DC fault occurs, the backward TW $V_{b}(x, s)$ induced from the fault point propagates towards terminal A. During the initial TW propagation stage, the detected backward TW at terminal A in $s$-domain is described as $V_{b}(0, s)$ :

$$
V_{b}(0, s)=e^{-\gamma(s) x} V_{b}(x, s)
$$

where $e^{-\gamma(s)}$ is the TW propagation coefficient. It can be expressed as [23]:

$$
e^{-\gamma(s) x} \approx \frac{1-k_{a} x / /}{\left(1+s T_{a}\right)} e^{-s x / v}
$$

where $e^{-s x / v}$ denotes the phase-change characteristics of TWs. $T_{a}$ is the dispersion time constant, which represents the TW distortion during the TW propagation. $T_{a}$ is highly dependent on fault distance, which is proportional to fault distance [23]. $k_{a}$ is the attenuation coefficient in per unit, which represents the attenuation effects of line resistance and line-to-ground conductance on TWs. $k_{a}$ can be written as:

$$
k_{a}=\operatorname{Re}\left[\sqrt{\left(r_{0}+j \omega L_{0}\right)\left(g_{0}+j \omega C_{0}\right)}\right]
$$

where $r_{0}$ and $L_{0}$ are per-unit line resistance and inductance respectively. $g_{0}$ and $C_{0}$ are per-unit line-to-ground conductance and capacitance of the distributed-parameter model of the transmission line respectively, as shown in Fig. 4 [21].

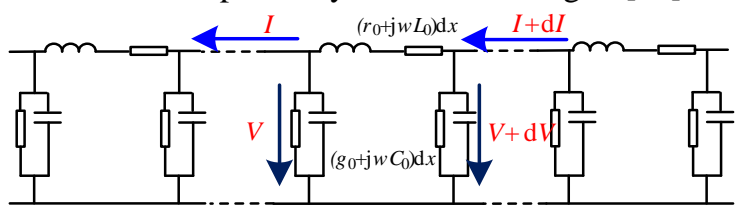

Fig. 4. The distributed-parameter model of the transmission line.

The diagram of measured initial backward TWs at terminal A under different fault distances is depicted in Fig. 5. Where $t_{0}$ is the time when DC fault occurs. $x_{1} / v$ and $x_{2} / v$ are the arrival times when backward TWs under different fault distances.

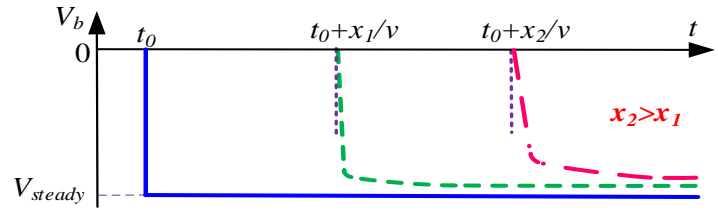

Fig. 5. Measured initial backward TWs at terminal A under different fault distances.

Based on the aforementioned analysis and Fig. 5, it can be concluded as follows:

(1) When the fault distance increases, the backward TW will arrive at point $\mathrm{A}$ later.

(2) Under remote DC faults, the magnitude of the backward TW is smaller, which demonstrates that a larger fault distance 
leads to the severer attenuation on the backward TW.

(3) With a larger fault distance, the time when the backward TW reaches the steady-state is longer. Thus, a larger fault distance will bring about a larger dispersion time constant $T_{a}$.

\section{B. The Analysis of Line-mode Backward TW}

Based on the phase-modal transformation, the backward TW $V_{b}$ can be decoupled into the line-mode backward TW $V_{b 1}$ and the zero-mode backward TW $V_{b 0}$. As pointed out by [21], the line-mode backward TW $V_{b 1}$ has a steady propagation speed, rarely depending on frequencies. Thus, the line-mode backward TW $V_{b 1}$ is adopted to highlight the propagation characteristics of TWs under different fault distances.

According to equations (7) and (8), the initial line-mode backward TW measured at terminal A can be written as:

$$
\begin{aligned}
V_{b l}(0, s) & =e^{-\gamma(s) x} V_{b l}(x, s)=\frac{1-k_{a} x / l}{\left(1+s T_{a}\right)} e^{-s x / v} V_{b I}(x, s) \\
& =\frac{k\left(1-k_{a} x / l\right)}{s\left(1+s T_{a}\right)} e^{-s x / v}
\end{aligned}
$$

where $k / s$ represents the amplitude of the initial line-mode backward TW at the fault point in $s$-domain. Using the reverse Laplace transform, the initial line-mode backward TW measured at terminal A can be expressed in time-domain as:

$$
V_{b l}(0, t)=V_{\text {steady_l }}\left(1-e^{-T_{a}\left(t-t_{0}-\frac{x}{v}\right)}\right) \varepsilon\left(t-t_{0}-\frac{x}{v}\right)
$$

where $t_{0}$ represents the time of the fault occurrence and $\varepsilon()$ represents the step function. The zoomed-in view of the measured line-mode backward TW $V_{b 1}$ is shown in Fig. 6, where $S_{1}$ and $S_{2}$ are the shadow areas marked with the oblique and horizontal lines respectively. They can be calculated as:

$$
\left\{\begin{array}{c}
S_{1}=\int_{\Delta t_{D F}} V_{b 1}(0, t) d t \\
S_{2}=V_{\text {steady_l }} \times \Delta t_{D F}-S_{1}
\end{array}\right.
$$

where $\Delta t_{D F}$ is the time interval when the initial backward TW $V_{b 1}$ reaches steady-state.

To evaluate the distortion effects of transmission lines on backward TWs (dispersion time constant $T_{a}$ ) under different fault distances, the distortion factor $(D F)$ is defined as:

$$
D F=\frac{S_{2}}{S_{1}+S_{2}}
$$

The distortion factor $(D F)$ represents the distortion effect of the transmission line on the initial backward TW. In the case of a larger fault distance, the distortion effect of the transmission line (the dispersion time constant $T_{a}$ ) is greater and the initial backward TW reaches steady-state later. Thus, the area $S_{l}$ is smaller while the area $S_{2}$ is larger, which results in a larger $D F$.

In addition, based on equations (11)-(13), the $D F$ can be calculated as:

$$
D F \approx 1-e^{-T_{a} \Delta t_{D F}}
$$

Equation (14) indicates that a larger dispersion time constant $T_{a}$ brings about a larger distortion factor $(D F)$. Since the larger fault distance brings about a larger $T_{a}$, there exists a positive correlation between the distortion factor $D F$ and fault distance $x$.

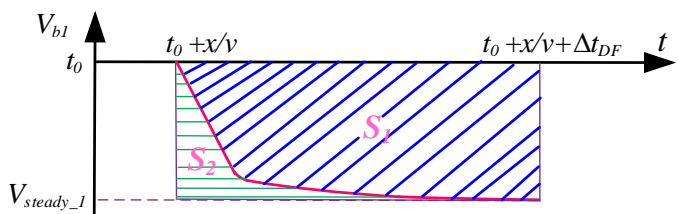

Fig. 6. The diagram of the distortion factor of line-mode backward TW.

To be concluded, the distortion factor $D F$ can be employed to identify faults under different fault locations, which provides a potential approach to detect internal and external faults.

\section{The Analysis of Zero-mode Backward TW}

Fig. 7 (a) shows the diagram of a positive PTG (P-PTG) fault, where $V_{f P}$ and $V_{f N}$ are the positive and negative PTG voltages at the fault point respectively. Using the phase-modal transformation matrix $Q$ [4], the line-mode and zero-mode voltages $\left(V_{f l}\right.$ and $\left.V_{f 0}\right)$ at fault point can be obtained:

$$
\left[\begin{array}{c}
V_{f 1} \\
V_{f 0}
\end{array}\right]=Q\left[\begin{array}{c}
V_{f P} \\
V_{f N}
\end{array}\right]=\frac{1}{2}\left[\begin{array}{cc}
1 & -1 \\
1 & 1
\end{array}\right]\left[\begin{array}{c}
V_{f P} \\
V_{f N}
\end{array}\right]
$$

The boundary condition under P-PTG fault is: $V_{f P}=0, I_{f N}=0$. Based on phase-modal transformation, the boundary condition can be written in modal domain as: $V_{f I}+V_{f 0}=0, I_{f l}=I_{f 0}$. The boundary condition indicates that the zero-mode circuit is connected to the line-mode circuit in series, as shown in Fig. 7 (b). Thus, $V_{f 0}$ can be obtained as:

$$
V_{f 0}=-V_{d c n} Z_{0} / 2\left(Z_{0}+Z_{1}+2 R_{f}\right)
$$

where $Z_{l}$ and $Z_{0}$ are line-mode and zero-mode impedances of the transmission line respectively. $V_{d c n}$ is the rated DC voltage and $R_{f}$ is the fault resistance. Whereas, under normal state, $V_{f P}$ is $V_{d c n} / 2$ and $V_{f N}$ is $-V_{d c n} / 2$. Thus, the zero-mode voltage $V_{f 0 \_n o r m a l}$ under normal state can be obtained as: $V_{f 0 \_ \text {normal }}=0$.

Thus, the superimposed equivalent voltage $V_{b 0}$ (zero-mode backward TW) at fault point can be expressed as:

$$
V_{b 0}=V_{f 0}-V_{f 0 \_n o r m a l}=-V_{d c n} Z_{0} / 2\left(Z_{0}+Z_{1}+2 R_{f}\right)
$$

Equation (17) indicates that the zero-mode backward TW $V_{b 0}$ is negative under P-PTG faults. Based on the aforementioned analysis in the modal domain, the zero-mode backward TW $V_{b o}$ under PTP faults and negative PTG faults (N-PTG) can also be obtained as:

$$
\left\{\begin{array}{cc}
V_{b 0}=V_{d c n} Z_{0} / 2\left(Z_{0}+Z_{1}+2 R_{f}\right) & (N-P T G \text { fault }) \\
V_{b 0}=0 & (P T P \text { fault })
\end{array}\right.
$$

As can be seen, the zero-mode backward TWs $V_{b 0}$ are positive under N-PTG faults while equal to zero under PTP faults, which demonstrates that the polarities of zero-mode backward TWs can be employed to select the faulty pole.

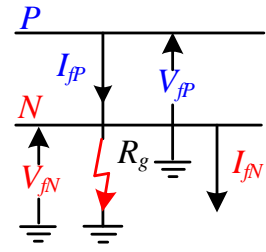

(a) A positive PTG fault

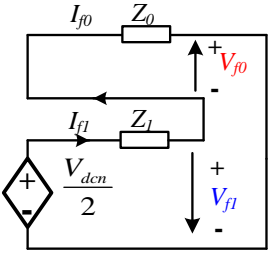

(b) Modal circuit
Fig. 7. The diagram of P-PTG fault and the composite modal network.

\section{The OVERALl Design OF THE DC FAUlt PROTECTION SCHEME INDEPENDENT OF BOUNDARY COMPONENTS}

A. The Fault Detection Criterion Based on Distortion Factor 
Fig. 8 shows the diagram of DC faults under different fault locations, where $F_{12}$ is an internal fault while $F_{1}$ and $F_{2}$ are external faults. Based on the aforementioned analysis, shorter fault distance results in a smaller $D F$. Thus, an internal fault can be identified as:

$$
D F(x) \leq D F(100 \%)
$$

where $D F(100 \%)$ is a measured $D F$ under the internal fault that is applied at the opposite terminal of the transmission line $F_{12}(100 \%)$.

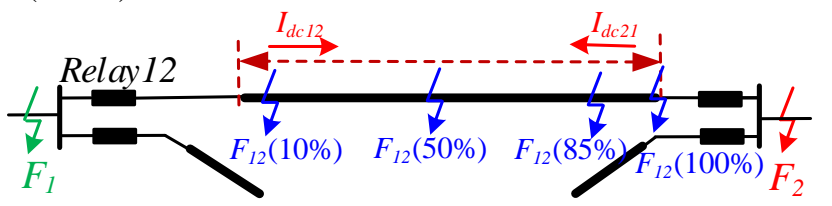

Fig. 8. DC faults under different fault distances.

However, due to the weak boundary effects provided by small CLRs, it is difficult to distinguish the internal fault at the end of the transmission line $\left(F_{12}(100 \%)\right)$ from the external fault $F_{2}$. To avoid mal-operation of relay 12 under external faults, the fault detection criterion depicted in equation (19) can be revised as:

$$
D F(x) \leq D F\left(\lambda_{\text {set }}\right)
$$

where $D F\left(\lambda_{\text {set }}\right)$ is a measured $D F$ under the internal fault whose fault distance is $\lambda_{\text {set }} \times l$. In AC protection schemes, the distance protection Zone $I$ is required to protect $80 \% \sim 90 \%$ of the entire transmission line [24]. Likewise, to achieve the above principle, $\lambda_{\text {set }}$ should satisfy:

$$
80 \% \leq \lambda_{\text {set }}<90 \%
$$

Equation (21) indicates that the proposed fault detection criterion cannot be employed to identify remote faults. According to the aforementioned speediness analysis of pilot protections, for remote faults, the detection time differences between pilot protection and single-end protection are small. Thus, pilot protection can be adopted to detect remote faults.

To be concluded, the internal fault detection criterion can be designed as:

$$
\left\{\begin{array}{cl}
D F\left(\lambda_{\text {set }}\right) / D F(x) \geq 1 & \left(0 \leq x / l \leq \lambda_{\text {set }}\right) \\
\text { Pilot Protection } & \left(\lambda_{\text {set }} \leq x / l \leq 1\right) \text { Zone II }
\end{array}\right.
$$

With regard to the design of the Zone II primary protection, pilot protection based on directional overcurrent in [20] is adopted to detect remote faults:

$$
\int_{\Delta t_{I d}} \Delta I_{m n}>0 \text { and } \int_{\Delta t_{I d}} \Delta I_{n m}>0
$$

where $\Delta I_{m n}$ and $\Delta I_{n m}$ are the changes of currents $I_{m n}$ and $I_{n m}$ that pass through the line $m n$. $\Delta t_{I d}$ is the data window for the integral of $\Delta I_{m n}$ and $\Delta I_{n m}$ and is selected as $0.1 \mathrm{~ms}$ in this paper.

\section{B. The Practicable Calculation of Distortion Factor DF}

To lower the computation burden and improve the computation speed, only some key points on the waveform of the voltage $V_{b l}$ are adopted to calculate the distortion factor DF, as shown in Fig. 9.

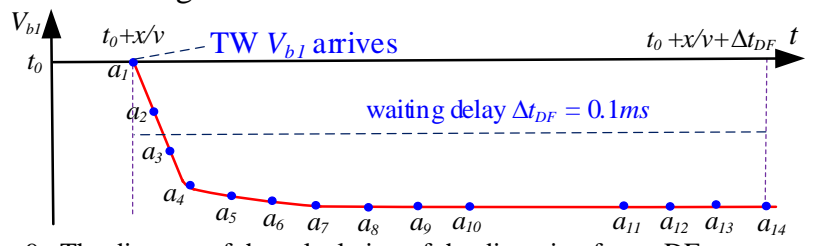

Fig. 9. The diagram of the calculation of the distortion factor DF.
In Fig. 9, $a_{1} \sim a_{10}$ are the ten successive sampling points with a sampling interval of $\Delta T_{s}$, of which the first point $a_{l}$ represents the arrival of line-mode backward TW. $a_{11} \sim a_{14}$ are four successive sampling points under steady-state. The time interval between points $a_{1}$ and $a_{14}$ is denoted as $\Delta t_{D F}$. To guarantee the accuracy of the practicable calculation, a narrow $\Delta T_{s}$ is adopted, which is $5 \mu \mathrm{s}$ in this paper. The backward TW $V_{b 1}$ reaches a steady-state within several sampling intervals. Considering a certain margin, $\Delta t_{D F}$ is selected as $0.1 \mathrm{~ms}$. Thus, the distortion factor DF can be approximated as:

$$
\begin{aligned}
& D F=1-\frac{S_{I}}{S_{I}+S_{2}} \approx 1-\frac{\left[\sum_{i=1}^{10} V_{b I}\left(a_{i}\right)+\sum_{i=11}^{14} V_{b l}\left(a_{i}\right)\right] \times \Delta t_{s}}{\frac{\sum_{i=11}^{14} V_{b I}\left(a_{i}\right)}{4} \times 14 \times \Delta t_{s}} \\
& =1-\frac{1}{14}\left(\frac{\sum_{i=1}^{10} V_{b l}\left(a_{i}\right)}{\frac{\sum_{i=11}^{14} V_{b l}\left(a_{i}\right)}{4}}-\frac{\sum_{i=11}^{14} V_{b l}\left(a_{i}\right)}{\sum_{i=11}^{14} V_{b l}\left(a_{i}\right)} 4\right)=\frac{5}{7}-\frac{1}{14} \times \frac{\sum_{i=1}^{10} V_{b l}\left(a_{i}\right)}{\sum_{i=11}^{14} V_{b l}\left(a_{i}\right)} \\
& =\frac{5}{7}\left(1-\frac{\sum_{i=1}^{10} V_{b 1}\left(a_{i}\right)}{\frac{10}{\sum_{i=11}^{14} V_{b 1}\left(a_{i}\right)}} 4\right)
\end{aligned}
$$

During the calculation of distortion factor $D F$, to determine the first point $a_{l}$ and the arrival time of line-mode backward $\mathrm{TW}$, a fault start-up element is employed. When criterion (25) holds true, the start-up element is activated:

$$
d V_{b 1} / d t \leq D_{\text {set }}
$$

where $D_{\text {set }}$ is the setting value of the fault start-up criterion.

\section{The Faulted Pole Selection Criterion based on Zero-mode Backward TW}

Based on the polarity analysis of zero-mode backward TW, the faulted pole selection criterion can be designed as:

$$
\begin{cases}V_{b 0}<-V_{\text {set }} & ; P-P T G \text { faults } \\ -V_{\text {set }}<V_{b 0}<V_{\text {set }} & ; P T P \text { faults } \\ V_{b 0}>V_{\text {set }} & ; N-P T G \text { faults }\end{cases}
$$

where $V_{\text {set }}$ is the positive threshold for faulted pole selection.

According to the aforementioned analysis, the overall DC fault protection scheme independent of boundary components can be obtained, as depicted in Fig. 10, where $D F\left(\lambda_{\operatorname{set} P(N)}\right)$ and $D F\left(\lambda_{\text {setd }}\right)$ represent the thresholds of $D F$ under PTG and PTP faults respectively. And $\Delta I_{m n P(N)}$ and $\Delta I_{n m P(N)}$ are the changes of the positive (negative) pole currents $I_{m n}$ and $I_{n m}$ of the line $m n$.

When the criterion (25) holds true, the fault start-up element will be activated and the subsequent protection scheme will be conducted. Firstly, zero-mode backward TW $V_{b 0}$ is employed to select the faulted pole. Then, the criterion (22) is adopted to identify internal and external faults. Once detecting $D F\left(\lambda_{\text {set }}\right) / D F \geq 1$, the fault is deemed to be internal. Otherwise, the pilot protection is used for further fault identification. A remote internal fault is not detected until the criteria $\int \Delta I_{m n P(N)}>0$ and $\int \Delta I_{n m P(N)}>0$ both hold true. Or else, the fault is 
identified to be external.

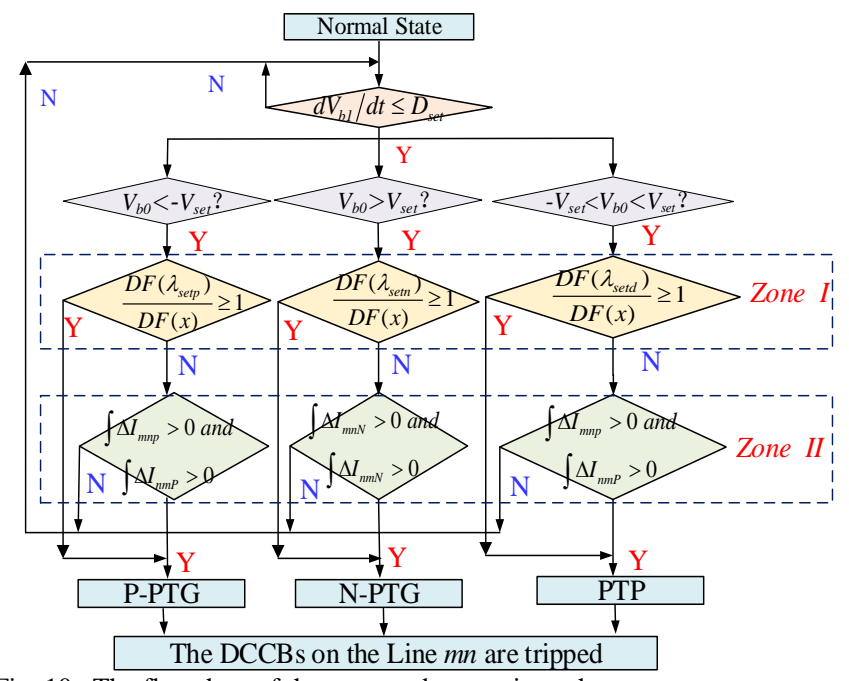

Fig. 10. The flowchart of the proposed protection scheme.

\section{SiMULATION AND VERIFICATION}

To verify the effectiveness of the proposed method, a $\pm 500 \mathrm{kV}$ four-terminal MMC based DC grid is built in PSCAD/EMTDC, as shown in Fig. 11. The CLR with $0.15 \mathrm{H}$ is implemented at the bus bar of each MMC, which is used to suppress the fault current rise but not to provide boundary effects for line protection. The system is a symmetrical monopole structure and the transmission line adopts the frequency-dependent overhead line (OHL) model. The length of each OHL is given in Fig. 11. The other converter parameters are listed in Table 1. MMC 3 controls the DC link voltage while other converters control the transmitted power.

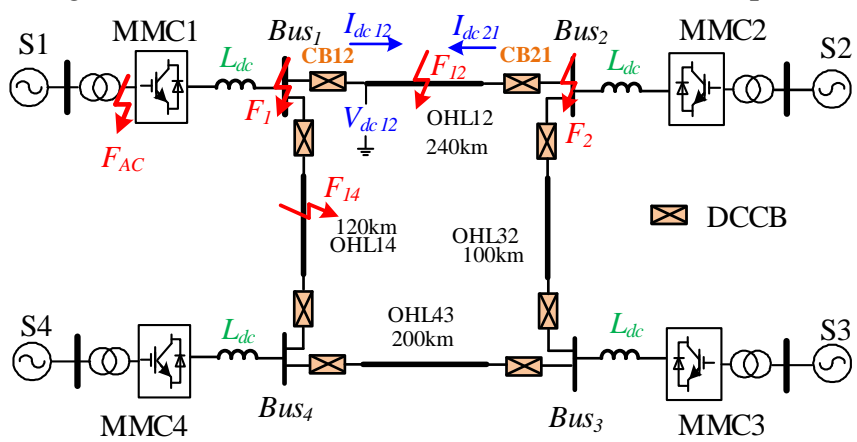

Fig. 11. The typology of a four-terminal MMC based DC grid. Table 1 Parameters of each converter station

\begin{tabular}{c||cccc}
\hline Converter & MMC1 & MMC2 & MMC3 & MMC4 \\
\hline arm inductance / mH & 50 & 50 & 50 & 50 \\
sub-module capacitor / $\mathrm{mF}$ & 4 & 4 & 8 & 8 \\
sub-module number / $N$ & 200 & 200 & 200 & 200 \\
rate power / MW & 750 & 750 & 1500 & 1500 \\
DC voltage $/ \mathrm{kV}$ & \pm 500 & \pm 500 & \pm 500 & \pm 500 \\
AC voltage $/ \mathrm{kV}$ & 380 & 380 & 380 & 380 \\
The SM voltage $/ \mathrm{kV}$ & 5 & 5 & 5 & 5 \\
\hline
\end{tabular}

\section{A. Determination of Threshold Setting}

Taking the relay of CB12 as an example, the threshold setting calculation will be conducted as follows.

\section{1) Threshold Setting $D_{\text {set }}$ for Fault Start-up Element}

According to criterion (25), the principle to select $D_{\text {set }}$ can be designed as follows:

The start-up element is aiming at identifying the faulted and normal states, rather than external and internal faults. As for the selectivity, the following protection algorithm will be adopted for further fault identification. Thus, $D_{\text {set }}$ should be activated during internal DC faults while not be activated under normal state. Thus, to cover all internal faults, $D_{V d c}$ (negative) should be larger than the observed maximum value of $d V_{b l} / d t$ under internal faults.

Based on the aforementioned analysis, various DC faults with different fault positions and fault resistances are applied to measure $d V_{b l} / d t$, as shown in Table 2.

Table 2 Measured $d V_{b l} / d t$ under different DC faults $(\mathrm{kV} / \mathrm{ms})$

\begin{tabular}{c|c||c|c|c|c}
\hline \multirow{2}{*}{ Fault distance } & \multirow{2}{*}{$\begin{array}{c}F_{l} \text { (Extern } \\
\text { al Faults) }\end{array}$} & $\begin{array}{c}\text { O\% of } \\
\text { Fault Resistance }\end{array}$ & & $50 \%$ of & $100 \%$ of \\
OHL12 & OHL12 & OHL12 \\
\hline \hline \multirow{2}{*}{ PTP } & $0.01 \Omega$ & -1377 & -2522 & -2257 & -2104 \\
\cline { 2 - 6 } & $300 \Omega$ & -785 & -1389 & -1247 & -1153 \\
\hline \multirow{2}{*}{ P-PTG } & $0.01 \Omega$ & -590 & -1047 & -918 & -847 \\
\cline { 2 - 6 } & $300 \Omega$ & -258 & -443 & -396 & -363 \\
\hline
\end{tabular}

From Table 2, it can be concluded that larger fault distance and fault resistance will lead to a decrease of amplitudes of $d V_{b l} / d t$. And the $d V_{b l} / d t$ under PTG faults have smaller amplitudes.

Hence, the maximum $d V_{b l} / d t$ (negative) under internal faults is $-363 \mathrm{kV} / \mathrm{ms}$, which can be obtained under the P-PTG DC fault at the end of OHL12 with $300 \Omega$ resistance. Based on the above principle, $D_{\text {set }}$ should be smaller than $-363 \mathrm{kV} / \mathrm{ms}$ and is selected as $-200 \mathrm{kV} / \mathrm{ms}$.

\section{2) Threshold Setting $V_{\text {set }}$ for Faulted Pole Selection}

According to equation (26), $V_{b 0}$ should be much smaller than $-V_{\text {set }}$ for P-PTG faults but much larger than $V_{\text {set }}$ for N-PTG faults. Based on the above analysis, applying various P-PTG faults with different fault distances and fault resistances on the overhead line OHL12, the voltages $V_{b 0}$ are shown in Fig.12.

As can be seen in Fig.12, a larger fault distance and fault resistance will bring about the smaller amplitude of the voltage $V_{b 0}$. Hence, the minimum value of the voltage $V_{b 0}$ can be obtained by the simulation of a P-PTG fault with $300 \Omega$ resistance at the end of OHL12, which is $116 \mathrm{kV}$. When the margin is selected to be $0.5, V_{\text {set }}$ can be calculated as: $V_{\text {set }}$ $=0.5 \times 116 \mathrm{kV}=58 \mathrm{kV}$.

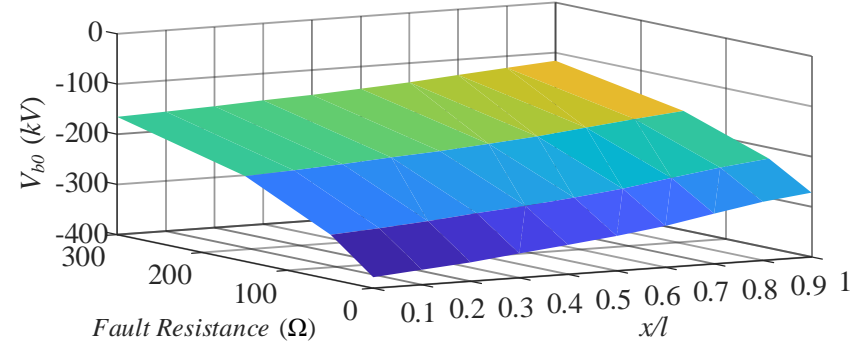

Fig. 12. The voltages $V_{b 0}$ under different fault locations and fault resistances.

\section{3) Threshold Setting $\lambda_{\text {setd( }(p)}$ and $D F\left(\lambda_{\text {setd( }(p)}\right)$ for Fault Detection}

According to the aforementioned analysis, the principle to select $D F\left(\lambda_{\text {setd }(p)}\right)$ is presented as follows:

On one hand, the proposed Zone I primary protection should protect the transmission line at least $80 \%$ of the entire length with $300 \Omega$ fault resistance. On the other hand, the proposed method should discriminate the metallic bus fault $F_{2}$ with a large margin. 
The distortion factor $D F$ will increase with the increase of fault distance and fault resistance. Compared with the fault distance, the impacts of fault resistance are smaller. Hence, PTG and PTP faults with $150 \Omega$ resistance at $85 \%$ of OHL12 are applied to obtain $D F\left(\lambda_{\text {setp }(n)}\right)$ and $D F\left(\lambda_{\text {setd }}\right)$ respectively. The simulation values under PTG and PTP faults are 0.0498 and 0.05171 respectively. Thus, $D F\left(\lambda_{\text {setp }(n)}\right)$ and $D F\left(\lambda_{\text {setd }}\right)$ are selected to be 0.0498 and 0.05171 respectively.

\section{B. Simulation Analysis for Internal Faults}

\section{1) Response to P-PTG Faults}

At 2.0s, a P-PTG fault with $0.01 \Omega$ fault resistance is applied at $25 \%$ of OHL12 and the simulation waveforms are shown in Fig. 13. As can be seen in Fig. 13(a), at 2.0002s, the backward TW arrives at the terminal of OHL12 and the $d V_{b 1} / d t$ is smaller than $-200 \mathrm{kV} / \mathrm{ms}$, which triggers the fault start-up element. In Fig. 13(b), $V_{b 0}<-V_{\text {set }}\left(-58 k V^{*} m s\right)$. Thus, it is deemed to be a PTG fault. $D F$ is 0.0111 and $D F\left(\lambda_{\text {setp }}\right) / D F$ is calculated as 4.4886, which satisfies the criterion (22), as shown in Fig. 13(c). Hence, it is deemed to be an internal fault. 2) Response to PTP Faults

At 2.0s, a metallic PTP fault happens at 25\% of OHL12 and the simulation results are shown in Fig. 14. In Fig. 14 (a), $d V_{b l} / d t<-200 \mathrm{kV} / \mathrm{ms}$, thus, the start-up element is activated. In Fig. 14 (b) and Fig. 14 (c), $\left|V_{b o}\right|<V_{\text {set }}$, and $D F\left(\lambda_{\text {setp }}\right) / D F=$ $3.6105>1$. Hence, it is identified to be an internal PTP fault. And the detection time is within $0.307 \mathrm{~ms}$. To be concluded, the proposed method can be used to detect internal faults quickly.

\section{Simulation Analysis for External Faults}

An external P-PTG fault with $0.01 \Omega$ resistance at $F_{2}$ is applied at 2.0s to verify the selectivity of the proposed method. The simulation waveforms are shown in Fig. 15.

As can be seen in Fig. 15 (a), $V_{b 0}<-V_{s e t}\left(-58 k V^{*} m s\right)$, which demonstrates the occurrence of the P-PTG fault. In Fig. 15 (b), $D F\left(\lambda_{\text {setp }}\right) / D F$ is 0.9419 , which does not satisfy the criterion of the Zone I primary protection in (22). Meanwhile, in Fig. 15 (c), $\Delta I_{2 I}$ is negative, which does not satisfy the criterion of the Zone II primary protection in (22), either. Hence, the fault is deemed to be internal, which demonstrates that the proposed method can identify external faults selectively.

\section{Detection Time}

For Zone I primary protection, the total detection time $t_{d_{-} z o n e I}$

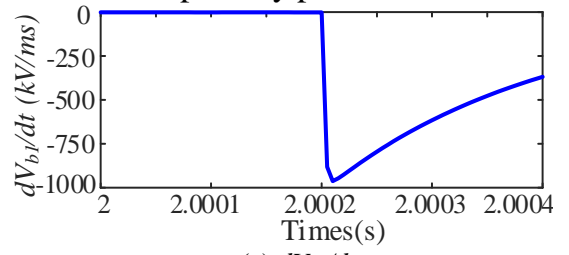

(a) $d V_{b 1} / d t$

Fig. 13. Simulation waveforms under a metallic P-PTG fault.

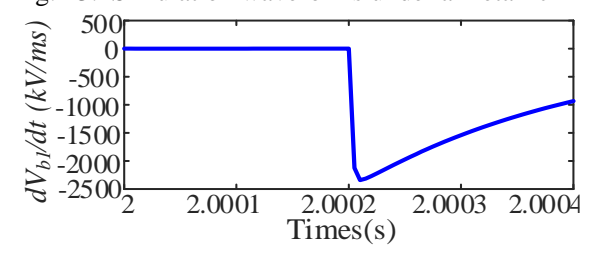

(a) $d V_{b 1} / d t$

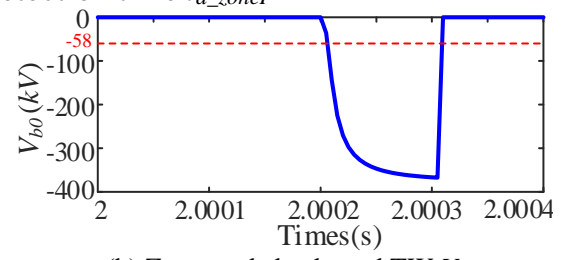

(b) Zero-mode backward TW $V_{b 0}$

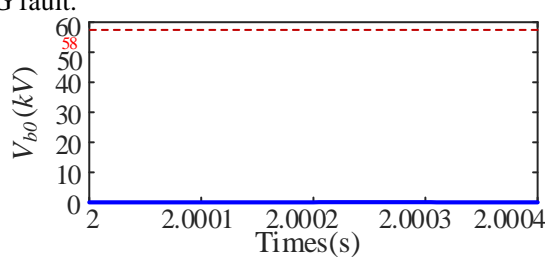

(b) Zero-mode backward TW $V_{b 0}$ mainly contains the traveling-wave propagation time, which has been introduced in Section II.B and the data window $\left(\Delta t_{D F}\right)$ for the calculation of DF. Thus, $t_{d_{-} z o n e l}$ can be approximated as:

$$
\begin{aligned}
t_{d_{-} \text {Zonel }} & =t_{d}(\text { single-end protection })+\Delta t_{D F} \\
& =\frac{x}{v_{T W}}+0.1 \mathrm{~ms}\left(0 \leq x \leq \lambda_{\text {set }} \times l\right)
\end{aligned}
$$

For the Zone II primary protection, the total detection time $t_{d_{-} z o n e I I}$ mainly contains the propagation time of the fault TW and the communication signal, and the data window $\left(\Delta t_{I d}\right)$ for the calculation of $\int \Delta I_{m n}$ and $\int \Delta I_{n m}$. Thus, $t_{d_{-} \text {zonelI }}$ can be approximated as:

$$
\begin{aligned}
t_{d_{Z} \text { ZonelI }} & =t_{d}(\text { pliot protection })+\Delta t_{I d} \\
& =\frac{l-x}{v_{T W}}+\frac{l}{v_{o f}}+0.1 m s\left(\lambda_{\text {set }} \times l \leq x \leq l\right)
\end{aligned}
$$

Considering that the Zone I primary protection can protect the $0 \sim 80 \%$ of the transmission line against different fault resistances, the maximum of $t_{d_{-} z o n e l}$ and $t_{d_{-} \text {zonell }}$ are respectively:

$$
\left\{\begin{array}{c}
t_{d_{-} Z \text { ZoneI }}=\frac{0.8 l}{v_{T W}}+0.1 \mathrm{~ms}=0.74 \mathrm{~ms} \\
t_{d_{-} \text {ZonelI }}=\frac{l-0.8 \mathrm{l}}{v_{T W}}+\frac{l}{v_{\text {of }}}+0.1 \mathrm{~ms}=1.46 \mathrm{~ms}
\end{array}\right.
$$

To be concluded, the longest detection time is approximately $0.74 \mathrm{~ms}$ for Zone I primary protection while $1.46 \mathrm{~ms}$ for Zone II primary protection.

\section{SENSITIVITY ANALYSIS}

The sensitivity analysis of the Zone II primary protection has been verified in [20]. Hence, only sensitivity analysis of the Zone I primary protection that is the main concern in this paper is conducted as follows.

\section{A. Robust to Fault Resistance}

Further studies are conducted to test the robustness of the proposed scheme against fault resistance. At 2.0s, PTP and P-PTG DC faults with different resistances varying from $0.01 \Omega$ to $300 \Omega(0.01 \Omega, 50 \Omega, 150 \Omega, 300 \Omega)$ are applied at $50 \%$ and $80 \%$ of OHL12 respectively. And the measured $D F\left(\lambda_{\text {setd }(p)) / D F}\right.$ are depicted in Fig. 17.

Fig. 14. Simulation waveforms under a metallic PTP fault.

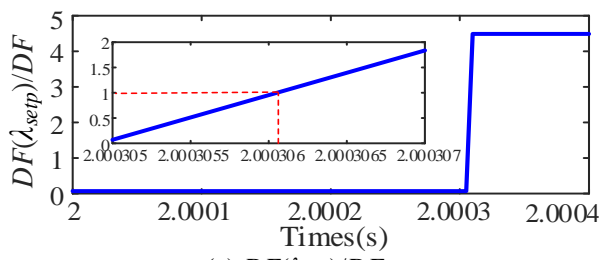

(c) $D F\left(\lambda_{\text {setd }}\right) / D F$

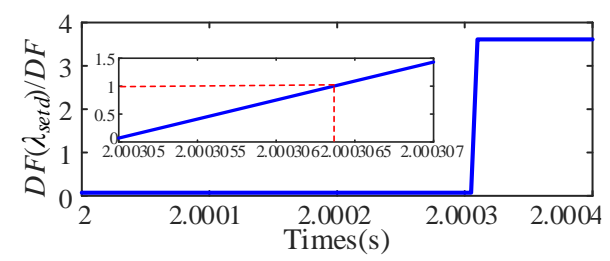

(c) $D F\left(\lambda_{\text {setd }}\right) / D F$ 


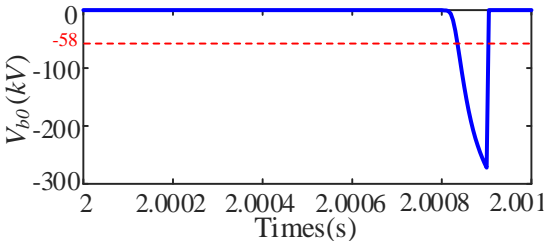

(a) Zero-mode backward TW $V_{b 0}$

Fig. 15. Simulation waveforms under an external metallic P-PTG fault.

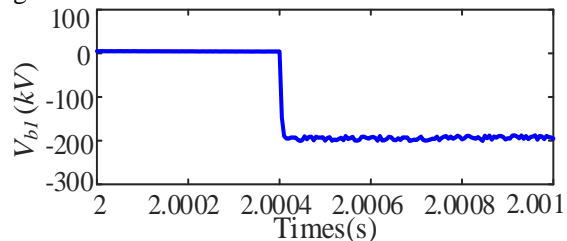

(a) line-mode backward TW $V_{b 1}$

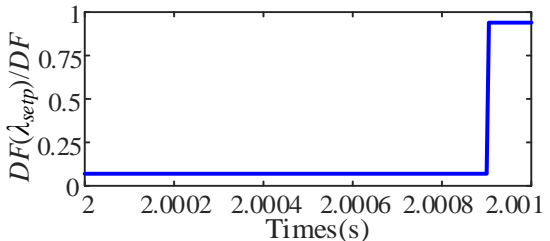

(b) $D F\left(\lambda_{\text {setp }}\right) / D$

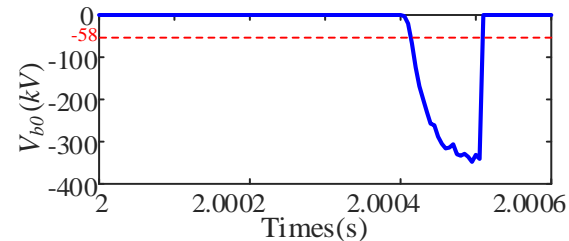

(b) Zero-mode backward TW $V_{b 0}$

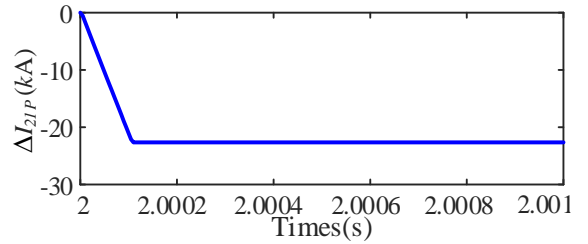

(c) $\Delta I_{21 P}$

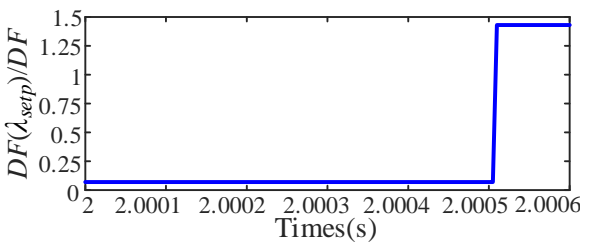

(c) $D F\left(\lambda_{\text {setd }}\right) / D F$

Fig. 16. Simulation waveforms under a P-PTG fault with $35 \mathrm{db}$ noise.

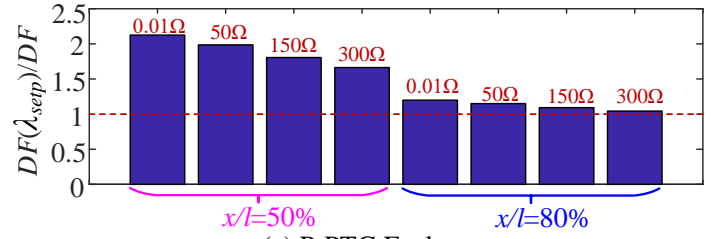

(a) P-PTG Faults

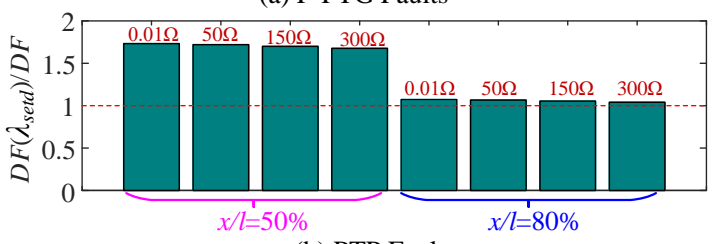

(b) PTP Faults

Fig. 17. $D F\left(\lambda_{\text {set }}\right) / D F$ under different faults with various fault resistances.

As can be seen in Fig. 17, under PTG faults, large fault resistance will increase $D F$, thereby resulting in $D F\left(\lambda_{\text {setp }}\right) / D F$ decreasing. However, even under faults with $300 \Omega$ fault resistance, fault detection criterion (22) still holds true, as shown in Fig. 17(a). In Fig. 17(b), under PTP faults, fault resistance has few impacts on $D F\left(\lambda_{\text {setd }}\right) / D F$. To be concluded, the proposed method is robust to fault resistance.

\section{B. Impacts of Fault Distance}

With fault distances varying from $24 \mathrm{~km}$ to $240 \mathrm{~km}$, PTP and P-PTG faults with $50 \Omega$ fault resistance are applied to test the impacts of fault distance on the proposed method and the measured $D F$ and $D F\left(\lambda_{\text {set }}\right) / D F$ are presented in Fig. 18.

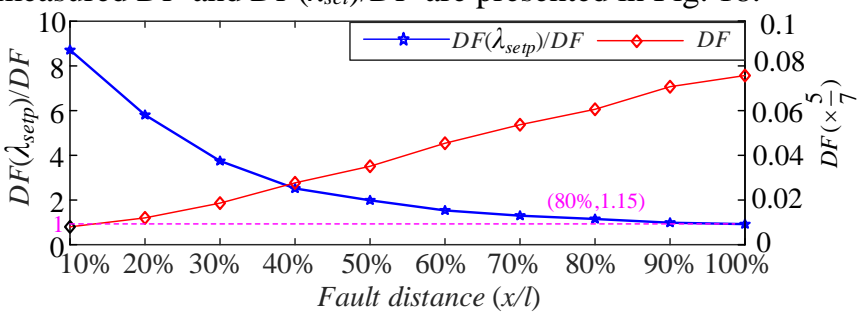

(a) P-PTG Faults

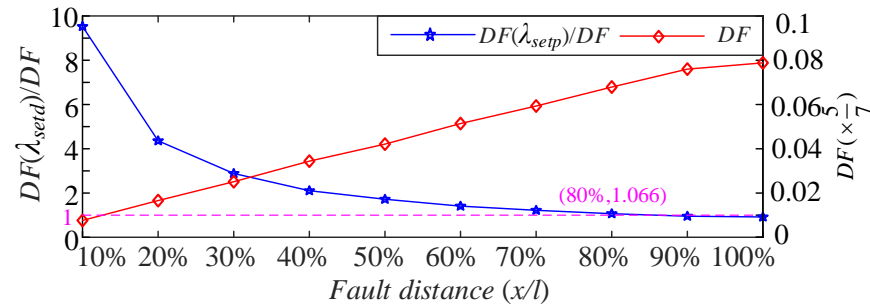

Fig. 18. The $D F$ and $D F\left(\lambda_{\text {set }}\right) / D F$ under DC faults with various fault distances.

\section{Impacts of Noise}

The white noise with $35 \mathrm{db}$ is added into the original line-mode backward TW $V_{b 1}$ and one-mode backward TW $V_{b 0}$ to test the impacts of noise, as shown in Fig. 16(a) and Fig. 16 (b). At $2 \mathrm{~s}$, a metallic P-PTG fault is applied in the middle of OHL12 and the simulation waveforms are shown in Fig. 16.

As can be seen in Fig. $16, V_{b 0}<-V_{\text {set }}$ and $D F\left(\lambda_{\text {setp }}\right) / D F>1$. Hence, it is identified to be an internal P-PTG fault, which demonstrates that the proposed method has a certain capability of endurance to noise.

\section{Response to AC Faults}

To test the impacts of AC faults on DC fault protection, at $2.0 \mathrm{~s}$, the metallic three-phase $\mathrm{AC}$ fault $F_{A C}$ is applied at the secondary side of the interface transformer close to MMC1. The simulation waveform is shown in Fig. 19.

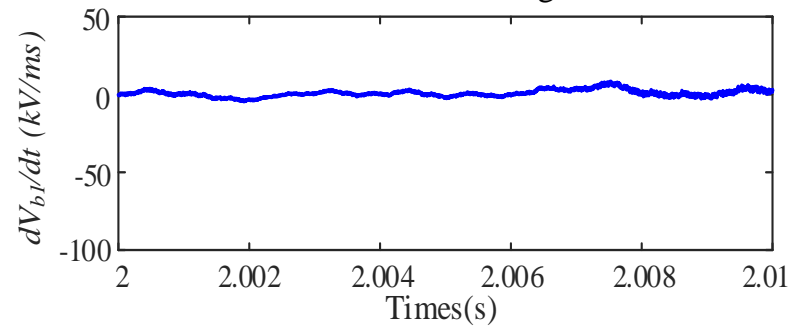

Fig. 19. The simulation waveforms under a metallic AC fault close to MMC1.

Under AC faults, no backward TW from fault point propagates towards the measured point. Thus, the magnitude of the change rate of line-mode backward TW $d V_{b l} / d t$ is much larger than $-200 \mathrm{kV} / \mathrm{ms}$, which will not trigger the fault start-up element, as shown in Fig. 19. Thus, AC faults have no impact on the proposed method.

\section{EVALUATION OF USING DIRECTIONAL OVERCURRENT BASEd PILOT PROTECTION AS THE ZONE II PRIMARY PROTECTION}

In Section II. B, the detection delay of pilot protection has been analyzed. The analysis results demonstrate that the extra time delay of pilot protection decreases when the fault distance increases. Besides, the rise speed of the fault current slows down when the fault distance increases, which leaves a larger detection time margin. Thus, it is reasonable to employ pilot protection to detect remote faults. The simulation results in 
Section V.D show that the detection time is no more than $1.5 \mathrm{~ms}$, satisfying the speediness requirement.

To further evaluate the rationality of using the directional overcurrent pilot protection as the Zone II primary protection, the communication reliability is analyzed. Considering that redundant primary protection schemes are typically required, redundant single-end protection schemes under weak boundary conditions are designed.

\section{A. The Communication Reliability Evaluation}

Firstly, compared with the current differential based pilot protection, no synchronous communication is required for the directional overcurrent based pilot protection. Thus, there is no synchronous delay and synchronous communication error. In addition, GPS systems are not required.

Secondly, optical fiber cables are adopted to communicate between converter stations. Due to the high reliability and strong anti-interference ability, optical fiber communication has been regarded as a highly reliable communication network for high-speed data transmission [25].

Lastly, two separate communication channels are implemented. One is adopted as the main channel while the other is adopted as an auxiliary channel. Under unfaulty states, the main channel will be checked by the monitoring system, so as to restore the normal state quickly. During the DC faults, if the main channel is out of work, the auxiliary channel works, thereby improving the communication reliability.

In addition, the pilot protection schemes have been applied in the Nan'ao $\pm 160 \mathrm{kV}$ three-terminal MMC-HVDC project as one of the primary protection schemes. In the Nan'ao project, $10 \mathrm{mH}$ CLRs are adopted as the boundary components. Under weak boundary conditions, reference [26] adopts the overcurrent protection, low-voltage protection together with the current differential protection to identify the internal and external faults. Obviously, due to smaller CLRs, even under low-impedance faults, overcurrent protection together with low-voltage protection cannot protect the entire transmission line. Thus, to detect remote faults and high-impedance faults, the pilot protection is adopted as one of the primary protection schemes, which is similar to the Zone II pilot protection in this paper. It demonstrates that pilot protection schemes can be adopted as a part of primary protection schemes in MMC-HVDC projects.

To be concluded, the directional overcurrent based pilot protection can be adopted as the Zone II primary protection with fast detection speed and high communication reliability.

\section{B. The Configuration of Redundant Single-end Protection Schemes Under Weak Boundary Conditions}

Under weak boundary conditions, the reliability of the single-end boundary protection schemes will be deteriorated. Particularly, when the CLRs are not implemented at line terminals, some boundary protection schemes cannot work, e.g. WT based methods in high-frequency domain [8][10], and CLR based methods [4][13][14]. Considering that fault TW amplitudes will decrease with the increase of fault distance, some time-domain TW based methods, such as ROCOV based protection, low-voltage criterion, overcurrent protection and so on, can still work well for close-in faults and low-impedance faults.

Compared with the low-impedance and close-in faults, high-impedance faults and remote faults will bring about smaller fault currents. Thus, low-impedance faults and close-in faults should be paid more attention to during the redundant protection scheme configuration. Taking the Siemens HVDC protection as an example, which has been applied in the Yun'guang UHVDC project [23], multiple redundant single-end fault detection criteria are adopted, including the voltage derivative $(d V / d t)$, the low-voltage criterion $(\Delta V)$, the current derivative $(d I / d t)$. However, when a remote PTG fault with $100 \Omega$ fault resistance happens, these redundant primary protection schemes refuse to trip [27]. Similarly, as mentioned above, in Nao'ao project, the overcurrent protection, low-voltage protection together with the current differential protection are adopted to achieve the configuration of redundant primary protection schemes.

As can be seen from the aforementioned project experience, for some single-end redundant primary protection schemes, they cannot protect the full transmission line against different fault resistances $(0 \sim 300 \Omega)$. The pilot protection schemes are still required to detect remote high-impedance faults, even medium-impedance faults. In addition, these redundant protection schemes are mainly adopted to improve the reliability of fault detection under close-in faults and low-impedance faults. Hence, the overcurrent protection and low-voltage protection together with the proposed scheme are adopted as the redundant primary protection schemes in this paper.

In addition, the DCCB protection is adopted as the back-up protection. Namely, when the fault current flowing through the DCCB exceeds the threshold, the DCCB will be tripped. Though the DCCBs on the healthy lines may also be tripped, the DCCB protection can avoid the MMC blocking and guarantee the power transmission of some healthy lines, thereby avoiding the shut-down of the entire DC grids [28].

To be concluded, the overcurrent protection, low-voltage protection together with the proposed scheme are adopted to achieve the configuration of redundant primary protection schemes. Moreover, the primary protection schemes together with back-up protection are adopted to guarantee the safe operation of DC grids.

\section{CONCLUSION}

To overcome the contradiction between weak boundary effects and reliability of fault protection, a fast and selective fault detection scheme independent of boundary components is proposed for MMC based DC grids in this paper. Based on the propagation characteristics analysis of TW, the distortion factor of the line-mode backward TW is employed as the Zone $I$ primary protection to identify internal and external faults. The polarity of zero-mode backward TW is employed to select faulted pole. In addition, pilot protection based on the directional overcurrent is adopted as the Zone II primary protection to identify internal remote faults.

1) In comparison with methods based on boundary effects, the proposed method operates effectively under weak 
boundary conditions. Meanwhile, assuming that the current-limiting effects provided by smaller CLRs can be guaranteed, the proposed protection method can decrease the size of CLRs, thereby lowering the cost and weight of CLRs.

2) Compared with the current differential based pilot protection, no synchronous communication is required for the directional overcurrent based pilot protection. In addition, two separate communication channels using optical fiber cables are implemented, which improves the communication reliability greatly. Aimed at detecting remote faults, the proposed pilot protection has a fast detection speed. The longest detection time is no more than $1.5 \mathrm{~ms}$.

3 ) The proposed method is endurable to high-resistance faults with $300 \Omega$ resistance and it is immune to noise with $35 \mathrm{~dB}$. Extensive simulation results demonstrate the method is robust to fault distances, fault types, and AC faults.

4) The overcurrent protection, low-voltage protection together with the proposed scheme are adopted to achieve the configuration of redundant primary protection schemes under weak boundary conditions. Moreover, the primary protection together with the back-up protection (DCCB protection) is adopted to guarantee the safe operation of DC grids.

\section{APPENDIX}

The overhead transmission lines are modeled based on the frequency-dependent (phase) model of PSCAD. Fig. 20 illustrates the configuration of DC overhead transmission line.

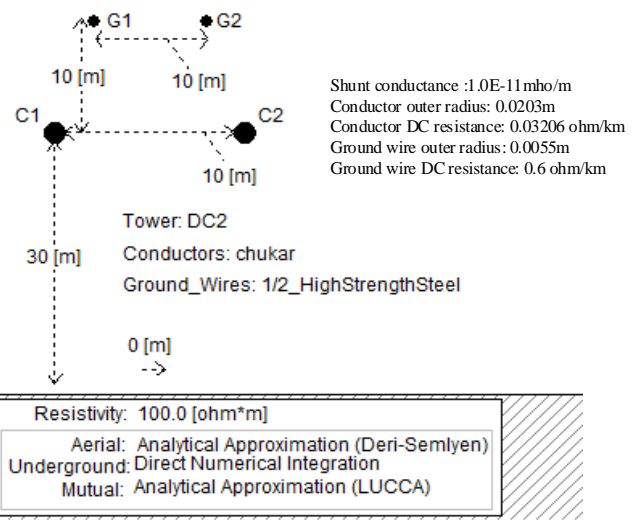

Fig. 20. Configuration of the overhead lines.

\section{REFERENCES}

[1] W. Xiang, S. Yang and J. Wen, "ANN-based robust DC fault protection algorithm for MMC high-voltage direct current grids," IET Renew. Power Gener., vol. 14, no. 2, pp. 199-210, Feb. 2020.

[2] S. Yang, W. Xiang, X. Lu, et al., "An Adaptive Reclosing Strategy for MMC-HVDC Systems With Hybrid DC Circuit Breakers," IEEE Trans. Power Del., vol. 35, no. 3, pp. 1111-1123, June 2020.

[3] M. Kong, X. Pei, H. Pang, et al., "A Lifting Wavelet-based Protection Strategy against DC Line Faults for Zhangbei HVDC Grid in China," presented in $19^{\text {th }}$ European Conf. on Power Electron. and Applica. (EPE'17 ECCE Europe), Warsaw, Poland, Sep. 2017, pp. 1-11.

[4] S. Yang, W. Xiang, R. Li, et al., "An Improved DC fault Protection Algorithm for MMC HVDC Grids based on Modal Domain Analysis," IEEE J. Emerg. Sel. Top. Power Electron., early access, to be published.

[5] J. Sneath, A. Rajapakse, "Fault Detection and Interruption in an Earthed HVDC Grid Using ROCOV and Hybrid DC breakers," IEEE Trans. Power Del., vol.31, no.3, pp. 973-981, Jun. 2016.

[6] N. Geddada, Y. M. Yeap, A. Ukil., "Experimental Validation of Fault Identification in VSC-based DC Grid System," IEEE Trans. Ind.
Electron., vol. 65, no. 6, pp. 4799-4809, June 2018.

[7] Q. Huang, G. Zou, X. Wei, et al., "A Non-unit Line Protection Scheme for MMC-based Multi-terminal HVDC grid," Int. J. Elect. Power Energy Syst., vol. 107, pp. 1-9, 2019.

[8] W. Xiang, S. Yang, L. Xu, et al., "A Transient Voltage based DC Fault Line Protection Scheme for MMC based DC Grid Embedding DC Breakers," IEEE Trans. Power Del., vol. 34, no. 1, pp. 334-345, Feb. 2019.

[9] L. Tang, X. Dong, S. Shi, et al., "A High-speed Protection Scheme for the DC Transmission Line of a MMC-HVDC Grid," Elect. Power Syst. Research, vol. 168, pp. 81-91, 2019.

[10] S. Zhang, G. Zou, Q. Huang, et al, "Single-ended Line Protection for MMC-MTDC Grids," IET Gener. Transm. Distrib., vol. 13, no. 19, pp. 4331-4338, Aug. 2019.

[11] B. Li, Y. Li, J. He, et al., "A Novel Single-Ended Transient-Voltage-Based Protection Strategy for Flexible DC Grid," IEEE Trans. Power Del., vol. 34, no. 5, pp. 1925-1937, Oct. 2019.

[12] S. Zhang, G. Zou, C. Wang, et al., "A Non-unit Boundary Protection of DC Line for MMC-MTDC Grids," Int. J. Elect. Power Energy Syst., vol. 116, pp. 1-9, 2019.

[13] R. Li, L. Xu, L. Yao, "DC Fault Detection and Location in Meshed Multi-terminal HVDC Systems based on DC Reactor Voltage Change Rate," IEEE Trans. Power Del., vol.32, no.3, pp. 1516-1626, Jun. 2017.

[14] C. Li, A. M. Gole, C. Zhao, "A Fast DC Fault Detection Method Using DC Reactor Voltages in HVdc Grids," IEEE Trans. Power Del., vol. 33, no. 5, pp. 2254-2264, Oct. 2018.

[15] D. Jovcic, W. Lin, S. Nguefeu, et al., "Low-Energy Protection System for DC Grids Based on Full-Bridge MMC Converters," IEEE Trans. Power Del., vol. 33, no. 4, pp. 1934-1943, Aug. 2018.

[16] M. A. Abdel-Moamen, S. A. Shaaban, F. Jurado, "France-Spain HVDC Transmission System with Hybrid Modular Multilevel Converter and Alternate-arm Converter," 2017 Innovations in Power and Advanced Computing Technologies (i-PACT), Vellore, 2017, pp. 1-6.

[17] X. Chen, H. Li, Y. Liang, et al., "A protection scheme for hybrid multi-terminal HVDC networks utilizing a time-domain transient voltage based on fault-blocking converters," Int. J. Elect. Power Energy Syst., vol. 118, pp. 105825, 2020.

[18] S. Yang, W. Xiang, J. Wen, "Analysis of Impact of Grounding-pole Current-limiting Reactor on Fault Detection of MMC based DC Grids," presented in $8^{\text {th }}$ Renewable Power Generation Conference (RPG 2019), Shanghai, China, Oct. 2019, pp. 1-6.

[19] G. Zou, Q. Feng, Q. Huang, et al., "A Fast Protection Scheme for VSC based Multi-terminal DC grid," Int. J. Elect. Power Energy Syst., vol. 98, pp. 307-314, 2018.

[20] S. Gao, G. Song, Z. Ma et al., "Novel Pilot Protection Principle for High-voltage Direct Current Transmission Lines based on Fault Component Current Characteristics," IET Gener. Transm. Distrib., vol. 9, no. 5, pp. 468-474, Feb. 2015.

[21] N. Tong, X. Lin, Y. Li, et al., "Local Measurement-Based Ultra-High-Speed Main Protection for Long Distance VSC-MTDC," IEEE Trans. Power Del., vol. 34, no. 1, pp. 353-364, Feb. 2019.

[22] N. Tong, X. Lin, C Li, et al., "Permissive pilot protection adaptive to DC fault interruption for VSC-MTDC," Int. J. Elect. Power Energy Syst., vol. 123, pp. 106234, 2020.

[23] C. Zhang, G. Song, T. Wang, et al., "Non-unit Traveling Wave Protection of HVDC Grids Using Levenberg-Marquart Optimal Approximation," IEEE Trans. Power Del. early access, to be published.

[24] S. Yang, W. Xiang, M. Zhou, et al., "A Single-end Protection Scheme for Hybrid MMC HVDC Grids Considering the Impacts of the Active Fault Current-limiting Control," IEEE Trans. Power Del. early access, to be published.

[25] M. Koshiba, K. Saitoh, Y. Kokubun, "Heterogeneous multi-core fibers: proposal and design principle," IEICE Electronics Express, 2009, vol.6, no.2, pp. 98-103, January 2009.

[26] Z. Guo, T. Liu, M. Chen, et al. "Isolation Strategy for Line Fault of Nan'ao Multi-Terminal VSC-HVDC Project." Southern Power System Technology, vol.12, no.2, pp. 41-46, 2018.

[27] H. Shu, X. Tian, J. Dong, et al. "Simulation and Analyses for Yun-Guang \pm 800 kV HVDC Transmission Line Protection System." Proceedings of the CSEE, vol.31, no.31, pp. 179-188, 2011

[28] W. Leterme, D. Van. Hertem. "Classification of Fault Clearing Strategies for HVDC Grids.” Cigre Lund Symposium 2015. 
This paper is a post-print of a paper submitted to and accepted for publication in IEEE Transaction on Power Delivery and is subject to

\section{BIOGRAPHIES}

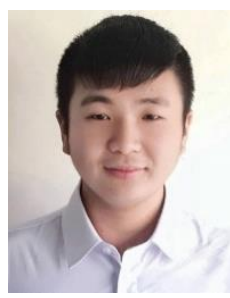

Saizhao Yang obtained his B.E. degree in electrical engineering from Huazhong University of Science and Technology (HUST), China, in 2018. He is currently pursuing his $\mathrm{PhD}$ degree at HUST. His research interests include dc fault protection of MMC-HVDC and dc grids.

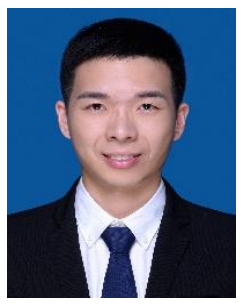

Wang Xiang (S'16-M'17) received his B.Eng. and $\mathrm{PhD}$ degrees both in electrical engineering from Huazhong University of Science and Technology (HUST), China in 2012 and 2017, respectively. He was a visiting student at the University of Aberdeen and the University of Strathclyde in 2014 and 2016 respectively. Currently, he is a research associate with the University of Strathclyde since 2018. His main research interests include MMC-HVDC, high power dc/dc converters and dc grids.

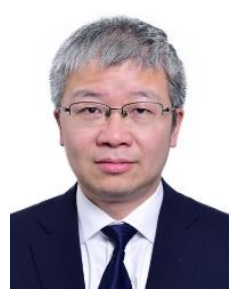

Jinyu Wen (M'10) received his B.Eng. and Ph.D degrees all in electrical engineering from Huazhong University of Science and Technology (HUST), Wuhan, China, in 1992 and 1998, respectively. He was a visiting student from 1996 to 1997 and a research fellow from 2002 to 2003 all at the University of Liverpool, UK, and a senior visiting researcher at the University of Texas at Arlington, USA in 2010. From 1998 to 2002 he was a director engineer in XJ Electric Co. Ltd. in China. In 2003 he joined the HUST and now is a Professor at HUST. His current research interests include renewable energy integration, energy storage application, DC grid, and power system operation and control. 